\title{
Complex and Robust Motion Performed in Extended Workspace with a Double Hexapod Robotic System
}

\author{
Mihai MĂRGĂRITESCU*, Dan DUMITRIU**, Cornel BRIŞAN***, Ana Maria Eulampia \\ ROLEA*, Anghel CONSTANTIN* \\ *National Institute of Research and Development in Mechatronics and Measurement Technique, Romania, Bucharest, \\ Sector 2, Sos. Pantelimon 6-8,021631,E-mail: mihai.margaritescu@gmail.com \\ **Institute of Solid Mechanics of the Romanian Academy, Romania, Bucharest, Sector 1, Str. Constantin Mille 15, 10141, \\ E-mail:dan.dumitriu@imsar.ro \\ ***Technical University of Cluj-Napoca, Romania, Cluj-Napoca, Str. Memorandumului 28, 400114, \\ E-mail: cornel.brisan@mdm.utcluj.ro
}

cross $^{\text {ref }}$ http://dx.doi.org/10.5755/j01.mech.26.6.20949

\section{Introduction}

The positioning systems with six degrees of freedom (DOFs) are used in different fields of activities such as advanced technologies or robotic surgery to obtain the desired position and orientation of an object in space. The most known such robots are the Gough-Stewart platforms, which are parallel structures characterized by high stiffness and high accuracy. The first application is mentioned in 1956 when Gough used it for testing tires. Stewart proposed in 1965 to be used as flight simulators, perhaps one of the most popular uses. For several decades, their direct and inverse kinematics were intense studied - Lee and Roth (1993), Raghavan (1993), Lazard and Merlet (1994), Husty (1996), Dietmaier (1998), G. Xiao-Shan, et al. (2005), together with calibration methods in order to improve the positioning accuracy. While the inverse kinematics is relatively simple, the direct kinematics is a very challenging problem due to the multiple solutions. Raghavan proved that "the Stewart platform of general geometry has 40 configurations" [1] and Dietmaier showed it can have 40 real postures [2].

On the other hand, the limited workspace is the main drawback of these parallel structures. The extension of the operating space can be achieved through the superposition of two or more hexapod platforms, obtaining staged structures with hybrid kinematics, parallel - serial kinematics. A common idea to solve this problem was to place the hexapod in the top of a serial robot or on other moving elements, but this solution is not applicable in tight spaces. The hybrid kinematics seems to be a possible solution. A multi hexapod robot produced by Logabex company is presented in book [3], this robot being extremely redundant, but difficult to be controlled. Consequently, the double hexapod idea is known, nevertheless it was not widely studied and implemented so far. Let us cite also Lee and Park [4, 5], who addressed the problematic issue of the direct kinematics of a double parallel robot arm, computing also the Jacobian for velocity control. They have also simulated a dynamics formulation derived from motor algebra and a virtual coefficient, computing the forces and moments acting at the passive joints as well as the active ones [4]. A workspace and singularity analysis was also performed, showing as expected the increased workspace of double hexapods and avoiding singularities by constraining the device motion [5].
A particular hybrid structure consists in two hexapods serially connected, having thus 12 DOFs, but this redundant structure is still controlled with difficulty. The idea developed on a previous own research project (2007 2010) was to use the double hexapod controlled so that the two hexapods have the same configuration any time in order to simplify the control. Different aspects regarding inverse kinematics of the double hexapod structure are treated in conference paper [6] at simulation level only. Related concerns, including dynamic aspects and intelligent control algorithms are reported more recently in the conference papers [7] and [8]. The HEXAGENT research project (Hexapod robotic system with extended mobility for intelligent actuation in limited spaces or hostile environments (20172018) has offered the opportunity to materialize the concept presented above and to test its performances.

\section{HEXAGENT demonstrator}

In this chapter the theoretical background, more precisely the inverse kinematics is presented, together with the preliminary modeling and simulation software. It follows the description of the system design, pointing the original solutions that lead to a supplementary extension of the robot workspace. The control software ends this section, being presented both the point to point (PTP) mode and the programmable mode, after a desired path.

\subsection{Inverse kinematics}

Three coordinate systems are attached to the three platforms of hexagonal shape. The first coordinate system $O_{0} x_{0} y_{0} z_{0}$ is attached to the geometrical center of the fixed base platform, where $O_{0}$ is the origin of this inertial system. The second moving system $O_{1} x_{1} y_{1} z_{1}$ is attached to the center of the intermediate platform 1 and the third moving system $O_{2} x_{2} y_{2} z_{2}$ is attached to the center of the upper platform 2 (Fig. 1).

To define the pose of a body solidary with a given point $P$, six parameters are used:

- three translations $x, y, z$;

- three independent angles (Euler's angles): $\psi$ is precession; - the rotation angle around the fixed vertical axis: $\theta$ is the nutation (tilt) angle and $\varphi$ is the intrinsic rotation (spin) angle around the vertical axes attached to the upper platform. 


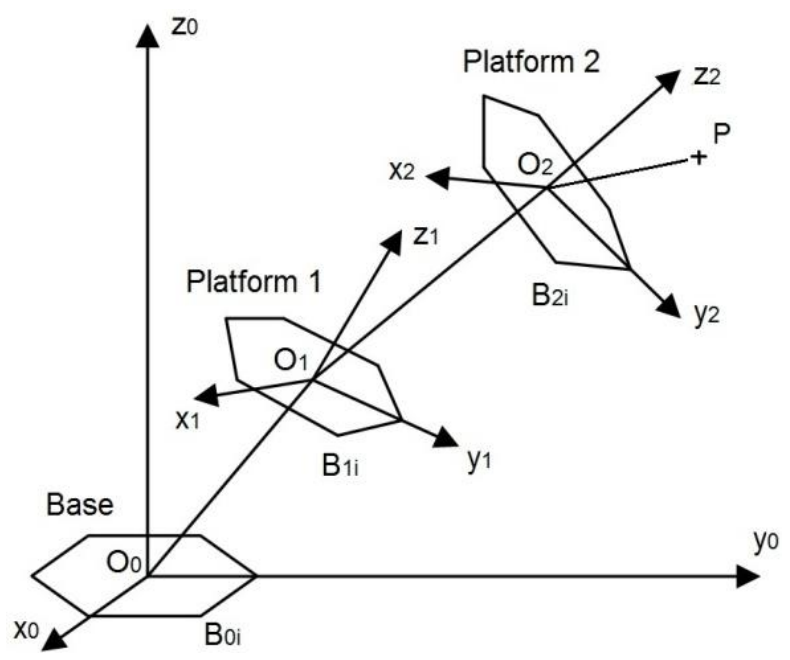

Fig. 1 Double hexapod coordinate systems

In order to establish the vertexes coordinates $B_{1 i}$ and $B_{2 i}, i=1, \ldots, 6$ of the hexagons, a matrices based method is used, with the following notations:

- the positioning vector:

$$
\boldsymbol{V}=\left[\begin{array}{l}
x \\
y \\
z
\end{array}\right],
$$

- the three elementary rotation matrices, corresponding to Euler's angles [9]:

$$
\begin{aligned}
\psi & =\left[\begin{array}{ccc}
\cos \psi & -\sin \psi & 0 \\
\sin \psi & \cos \psi & 0 \\
0 & 0 & 1
\end{array}\right], \\
\boldsymbol{\theta} & =\left[\begin{array}{ccc}
\cos \theta & 0 & \sin \theta \\
0 & 1 & 0 \\
-\sin \theta & 0 & \cos \theta
\end{array}\right], \\
\boldsymbol{\varphi} & =\left[\begin{array}{ccc}
\cos \varphi & -\sin \varphi & 0 \\
\sin \varphi & \cos \varphi & 0 \\
0 & 0 & 1
\end{array}\right] .
\end{aligned}
$$

Starting from the position and the orientation of the platform 2 - as input values -, described by the coordinates $x_{O_{2}}^{0}, y_{O_{2}}^{0}, z_{O_{2}}^{0}$ in the frame $O_{0} x_{0} y_{0} z_{0}$ and the orientation angles $\psi_{2}^{0}=\Psi, \theta_{2}^{0}=\Theta, \varphi_{2}^{0}=\Phi$ in the same frame, the analogue values are determined:

- $x_{O_{1}}^{0}, y_{O_{1}}^{0}, z_{O_{1}}^{0}$, respectively $\psi_{1}^{0}, \theta_{1}^{0}, \varphi_{1}^{0}$ in the frame $O_{0} x_{0} y_{0} z_{0}$

- $x_{O_{2}}^{1}, y_{O_{2}}^{1}, z_{O_{2}}^{1}$, respectively $\psi_{2}^{1}, \theta_{2}^{1}, \varphi_{2}^{1}$ in the frame $O_{1} x_{1} y_{1} z_{1}$.

For a given point $P$ we have the next relations [10] between its coordinates in the systems $O_{0} x_{0} y_{0} z_{0}, O_{1} x_{1} y_{1} z_{1}$, respectively $\mathrm{O}_{2} x_{2} y_{2} z_{2}$ :

$$
\boldsymbol{V}_{P}^{0}=\boldsymbol{V}_{O_{1}}^{0}+\boldsymbol{\psi}_{1}^{0} \times \boldsymbol{\theta}_{1}^{0} \times \varphi_{1}^{0} \times \boldsymbol{V}_{P}^{1},
$$

$$
\begin{aligned}
& \boldsymbol{V}_{P}^{1}=\boldsymbol{V}_{O_{2}}^{1}+\boldsymbol{\psi}_{2}^{1} \times \boldsymbol{\theta}_{2}^{1} \times \varphi_{2}^{1} \times \boldsymbol{V}_{P}^{2}, \\
& \boldsymbol{V}_{P}^{0}=\boldsymbol{V}_{O_{2}}^{0}+\boldsymbol{\Psi} \times \boldsymbol{\Theta} \times \boldsymbol{\Phi} \times \boldsymbol{V}_{P}^{2} .
\end{aligned}
$$

For identical configurations, imposed by the supplementary condition mentioned above, we have identical distances $O_{0} O_{1}=O_{1} O_{2}$, namely:

$$
\boldsymbol{V}_{O_{1}}^{0}=\boldsymbol{V}_{O_{2}}^{1},
$$

and identical angles:

$$
\left\{\begin{array}{l}
\boldsymbol{\psi}_{1}^{0}=\boldsymbol{\psi}_{2}^{1}=\boldsymbol{\psi} \\
\boldsymbol{\theta}_{1}^{0}=\boldsymbol{\theta}_{2}^{1}=\boldsymbol{\theta} \\
\boldsymbol{\varphi}_{1}^{0}=\boldsymbol{\varphi}_{2}^{1}=\boldsymbol{\varphi}
\end{array}\right.
$$

Combining Eqs. $(5-9)$, it is obtained the relation associated with the two identical hexapods structure [6]:

$$
\psi \times \boldsymbol{\theta} \times \boldsymbol{\varphi}=\sqrt{\Psi \times \Theta \times \Phi}
$$

Furthermore:

$$
\boldsymbol{V}_{O_{2}}^{0}=\boldsymbol{V}_{O_{1}}^{0}+\sqrt{\boldsymbol{\Psi} \times \boldsymbol{\Theta} \times \boldsymbol{\Phi}} \times \boldsymbol{V}_{O_{2}}^{1} .
$$

Using Eq. (8), we have:

$$
\boldsymbol{V}_{O_{2}}^{0}=(\boldsymbol{1}+\sqrt{\boldsymbol{\Psi} \times \boldsymbol{\Theta} \times \boldsymbol{\Phi}}) \times \boldsymbol{V}_{O_{1}}^{0},
$$

or

$$
\boldsymbol{V}_{O_{1}}^{0}=\boldsymbol{V}_{O_{2}}^{1}=(\boldsymbol{1}+\sqrt{\boldsymbol{\Psi} \times \boldsymbol{\Theta} \times \boldsymbol{\Phi}})^{-1} \cdot \boldsymbol{V}_{O_{2}}^{0}
$$

Thus, we can determine the coordinates of the points $B_{1 i}$ and $B_{2 i}, i=1, \ldots, 6$ :

$$
\boldsymbol{V}_{B_{1 i}}^{0}=\boldsymbol{V}_{O_{1}}^{0}+\sqrt{\boldsymbol{\Psi} \times \boldsymbol{\Theta} \times \boldsymbol{\Phi}} \times \boldsymbol{V}_{B_{1 i}}^{1},
$$

and

$$
\boldsymbol{V}_{B_{2 i}}^{1}=\boldsymbol{V}_{O_{2}}^{1}+\sqrt{\boldsymbol{\Psi} \times \boldsymbol{\Theta} \times \boldsymbol{\Phi}} \times \boldsymbol{V}_{B_{2 i}}^{2} .
$$

Replacing Eq. (13) in Eq. (14) and in Eq. (15), we obtain the final key relations:

$\boldsymbol{V}_{B_{1 i}}^{0}=(\boldsymbol{I}+\sqrt{\boldsymbol{\Psi} \times \boldsymbol{\Theta} \times \boldsymbol{\Phi}})^{-1} \times \boldsymbol{V}_{O_{2}}^{0}+\sqrt{\boldsymbol{\Psi} \times \boldsymbol{\Theta} \times \boldsymbol{\Phi}} \times \boldsymbol{V}_{B_{1 i}}^{1}$,

and

$\boldsymbol{V}_{B_{2 i}}^{1}=(\boldsymbol{I}+\sqrt{\boldsymbol{\Psi} \times \boldsymbol{\Theta} \times \boldsymbol{\Phi}})^{-1} \times \boldsymbol{V}_{O_{2}}^{0}+\sqrt{\boldsymbol{\Psi} \times \boldsymbol{\Theta} \times \boldsymbol{\Phi}} \cdot \boldsymbol{V}_{B_{2 i}}^{2}$

From the Eqs. (16) and (17), the lengths of the legs are easily deduced, namely the control parameters. 
As it is well known, the degree of mobility $M$ of the mechanism with $n$ mobile elements and having $N_{m}$ couples of $m$ class is:

$$
M=6 \cdot n-\sum_{m=1}^{5} m \cdot N_{m},
$$

which represents the number of independent parameters which determines its configuration. To establish it, the type of the kinematic joints must be defined.

According to the kinematic scheme (Fig. 2), each leg is linked by the lower and the upper platform using universal joints (Cardanic joints); between the linear actuator shaft, considered prismatic joint and the upper universal joint a radial bearing is placed, representing a rotation joint.

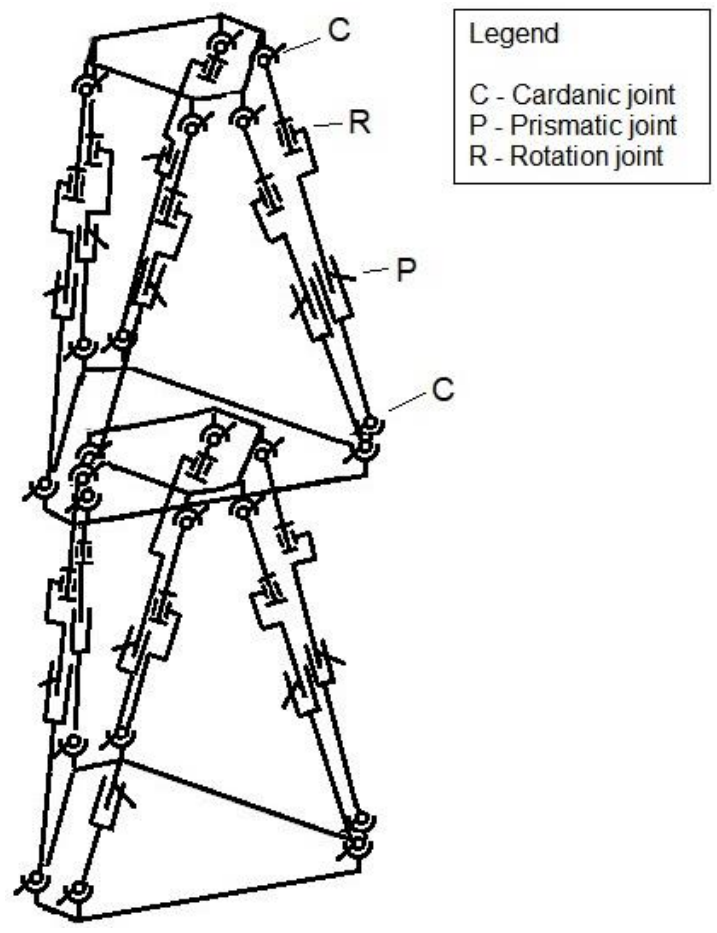

Fig. 2 Kinematic scheme

For one hexapod, the six legs consist of 18 elements and of the mobile platform (the $19^{\text {th }}$ element), namely $n=19$. In this case, there are:

-12 universal joint of class 4;

-6 translation joints of class 5 ;

-6 rotation joints of class 5 .

Thus, the degree of mobility of the hexapod in this construction has the expected value:

$$
M=6 \cdot 19-(4 \cdot 12+5 \cdot 6+5 \cdot 6)=6
$$

For the double hexapod, there are:

- 38 mobile elements;

-24 universal joint of class 4;

-12 translation joints of class 5 ;

-12 rotation joints of class 5 , resulting.

$$
M=6 \cdot 38-(4 \cdot 24+5 \cdot 12+5 \cdot 12)=12 .
$$

Due to the fact the system with 12 DOFs is very difficult to be controlled, it was imposed the condition that the two hexapods have the same geometrical configuration every moment. Thus, de double hexapod is software limited at 6 DOFs.

\subsection{Modeling and simulation}

In order to visualize and check the behavior of the double structure, a series of LabVIEW applications were developed. The main application (Fig. 3) contains the relations of the double hexapod inverse kinematics. The front panel comprises:

- top view of the double structure in the left-up corner;

- side view of the double structure in the right-up corner;

- dimensional/geometrical parameters of the platforms - the two radii and two angles that define the irregular hexagons and also the height of the characteristic point related to the upper platform - in the left-down corner.

- input values (external coordinates) and the output values (internal coordinates), more precisely the lengths of the legs in the right-down corner.

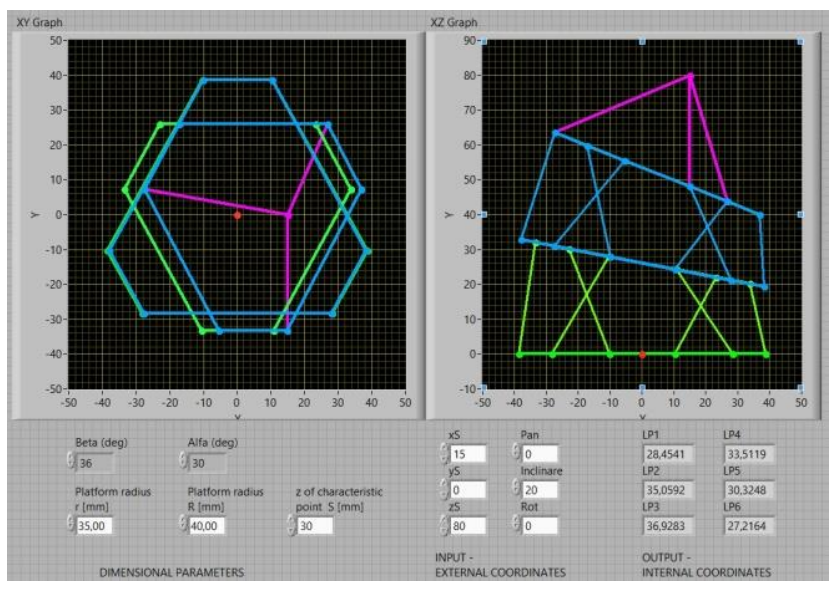

Fig. 3 Double hexapod modeling

The graphical code of the double hexapod inverse kinematics is too large to be presented in a single figure, but a small part dealing with implementation of the rotation matrices is shown (Fig. 4).

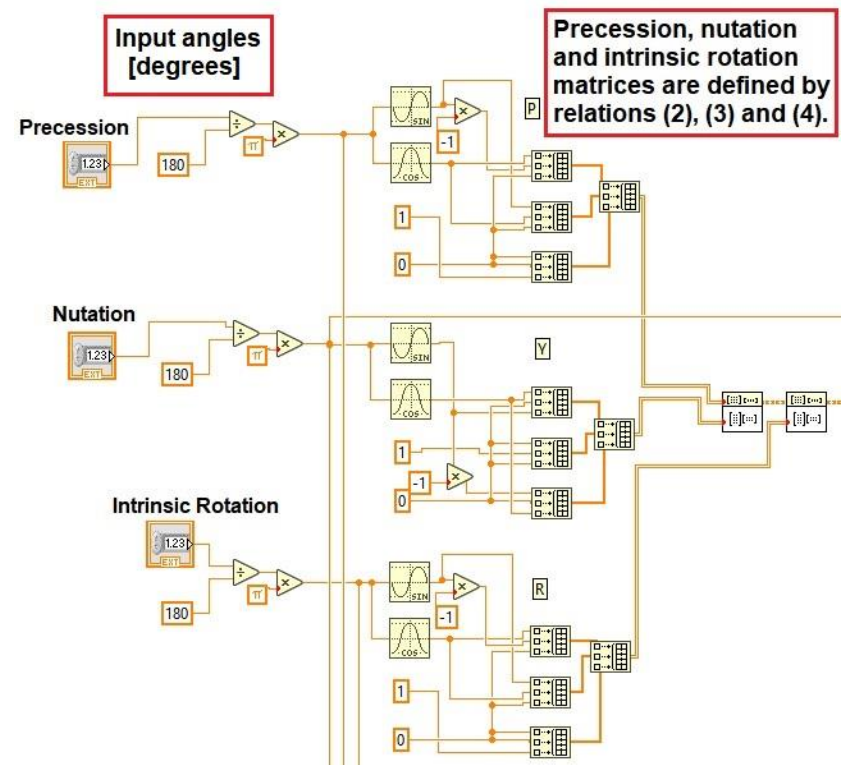

Fig. 4 Implementation of the elementary rotation matrices in LabVIEW 


\subsection{System design}

The HEXAGENT demonstrator structure (Fig. 5) is composed by the Double Hexapod (DHx) and the Electronic Control Module (ECM); The DHx comprises the linear actuators and the mechanical components. The ECM includes a control computer and two controllers with 6 channels; the Control Software (CSw) runs on ECM.

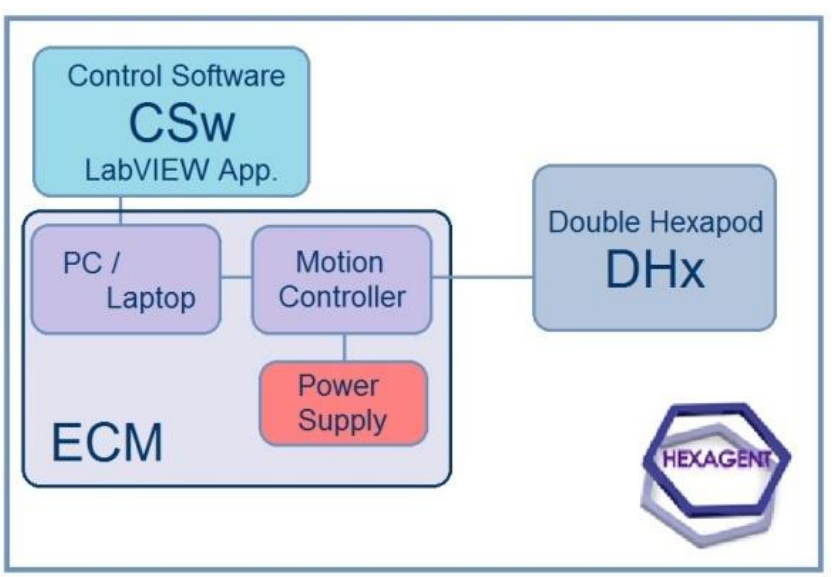

Fig. 5 System architecture

The system is based on two controllers C884K004, 6 channels type and 12 linear actuators L220.50DG type, both produced by Physik Instrumente (PI). Referring to the joints type, it is known that for one hexapod are necessary 6 spherical joints for one platform and 6 universal joints for the other, in order to obtain the mobility equal to 6 . For reasons of constructive simplicity, the HEXAGENT mechanical structure uses universal joints only; to assure the desired mobility, a supplementary rotation is introduced in every leg by the aid of a radial bearing. This construction, in the frame of the double hexapod system, is protected by the OSIM Romanian patent no. 125589/ 2016 [11]. The entire design is carried out in SolidWorks and most mechanical parts were obtained by the aid of the plastic Selective Laser Sintering (SLS) technology; the combination of them offered specific possibilities. The main idea was to build the bosses on which the universal joints are mounted inclined at angles that in the median position allow for an approximate alignment of the half-couplings. The design procedure of the intermediate platform with inclined bosses has specific features (Fig. 6). The geometric model represents the starting point of the physical model and the bosses are created first (Fig. 6, a), the platforms themselves being subsequent built (Fig. 6, b). This leads to a better use of the useful angle of the universal joints, having as result a supplementary extension of the robot workspace. This is facilitated by the use of SLS technology along with LabVIEW modeling that contains the entire geometry of the HEXAGENT system and also with the possibilities of the SolidWorks 3D design software that enables the materialization of complex geometric elements. The achievement of the fractional angles resulting from the geometry of the hexapod system seems difficult to be obtained both from the point of view of the designer and the technologist through classical methods, but it has proved realistic by the SLS technology. This solution also conducts to the elimination of some connecting elements, and thus contributes to the mounting errors reduction; it makes the object of the OSIM patent application no. A00112/ 2018.

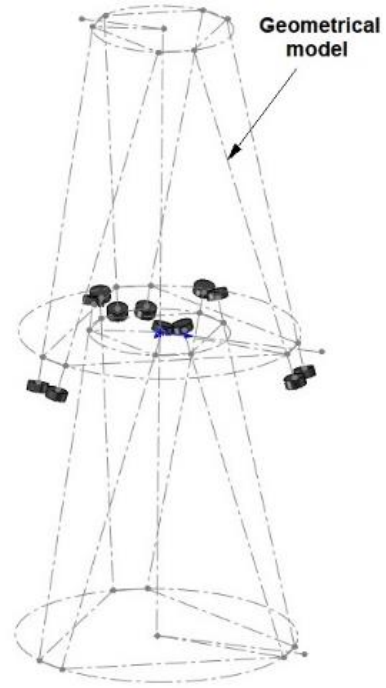

a

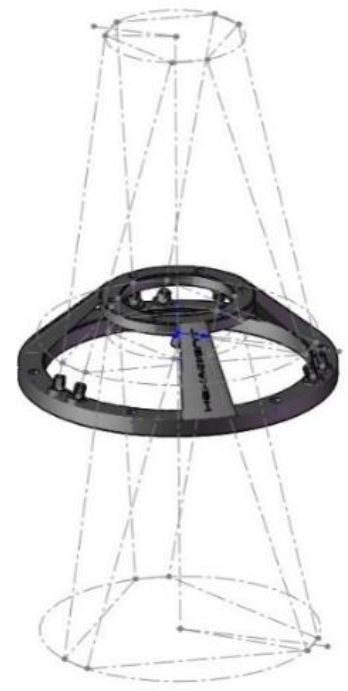

b
Fig. 6 Intermediate platform with inclined bosses: a Bosses design; $\mathrm{b}$ - final design

The linear module, containing the actuator and the radial bearing allows a roto-translational movement for the mobile terminal element and in the same time protects the actuator against the lateral forces. The complete system is shown for median (home) position (Fig. 7, a) and work position (Fig. 7, b).

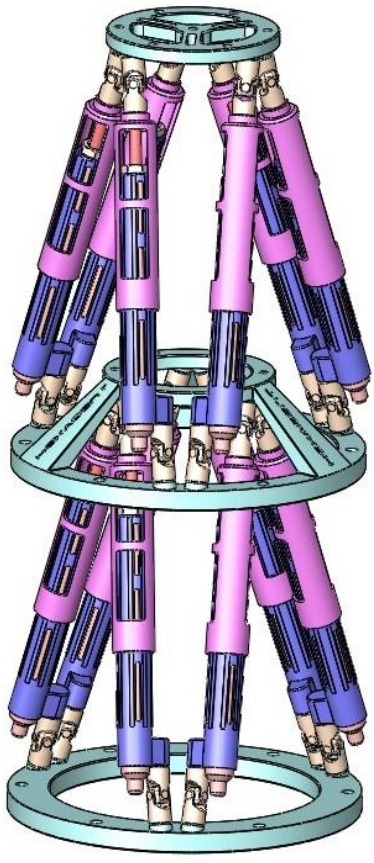

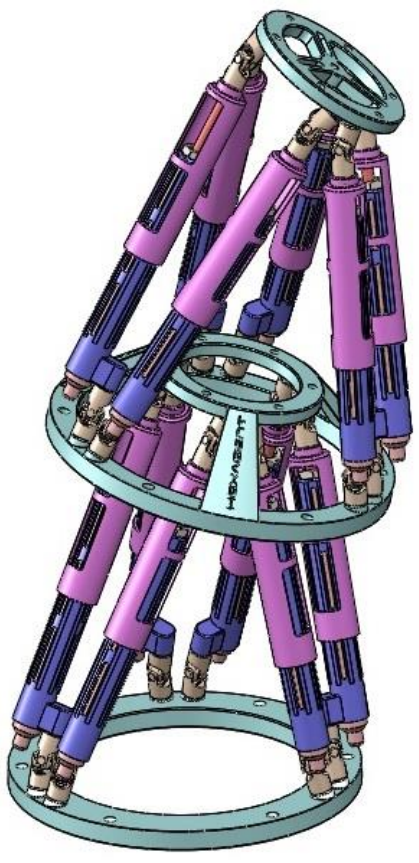

b
Fig. 7 Double hexapod system with alignment of the universal joints to the median position: a - home position, $\mathrm{b}$ - work position

The double hexapod is mounted on a frame made of aluminum profiles and Plexiglas walls with protective role (Fig. 8). 


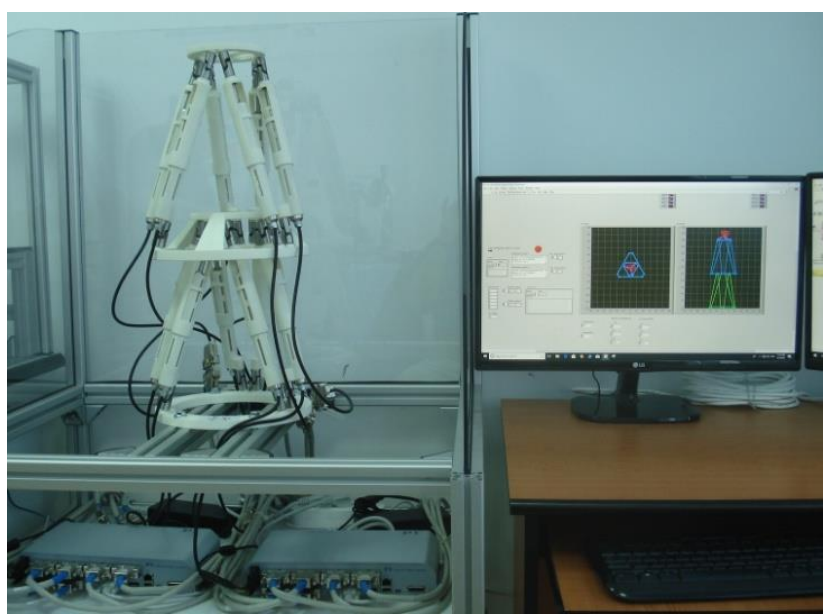

Fig. 8 HEXAGENT demonstrator set-up

\subsection{Control software}

The manual control software is of PTP type and is the basic way of controlling the double hexapod robotic system, being developed in the LabVIEW environment. It is based on the inverse kinematics relations of the HEXAGENT system, relationships that link the external coordinates of the robot (three independent translations and three independent rotations, - the 6 external DOFs at the characteristic point level) as inputs to the program and the internal coordinates - the linear actuator strokes. It should be noted that although there are 12 actuators, from the condition that the two hexapods have identical configurations every moment, a set of 6 program outputs result. The constructive parameters of the model were adapted to the actual sizes of the robot. In order to protect the actuators against possible damages at end of stroke, numeric controls were introduced so that to limit the linear motion in the desired range. If the robot receives a command exceeding the limits, the robot does not move or it returns to the home position if it works in a loop and at certain iteration one limit is also exceeded. This section represents the block diagram of the code, in which a subVI (a LabVIEW block) which comprises all inverse kinematics relations and the calculus of the constructive parameters was used (Fig. 9).

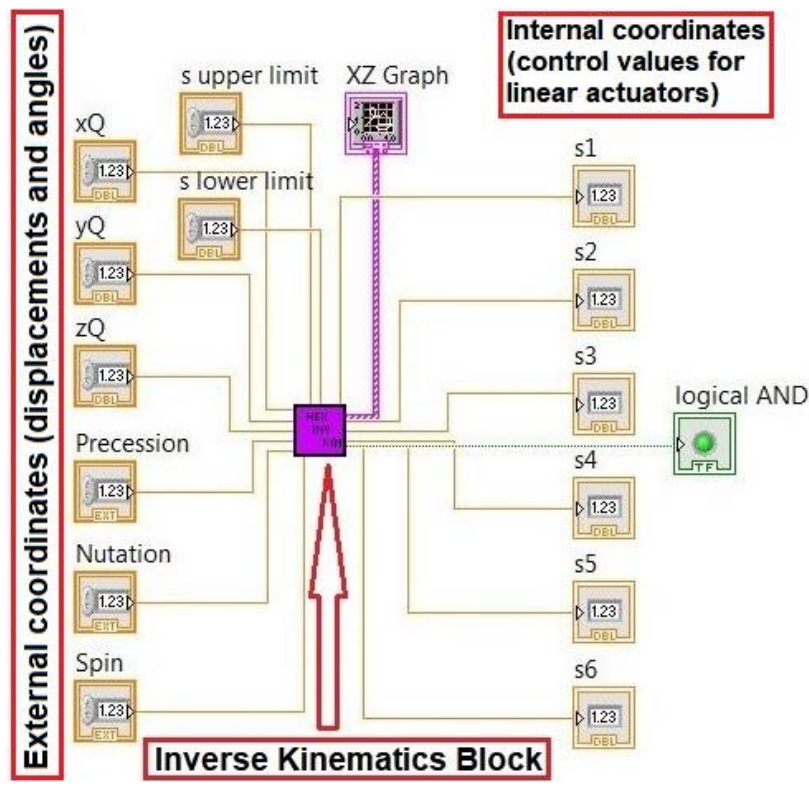

Fig. 9 Software of calculus of the control values
The control values are sent to controllers by the aid of several libraries provided by PI. This control application software, named HEXAGENT PTP Control, is shown as Control Panel (Fig. 10), containing the six controls associated with the desired pose and the two stroke limits in the left side, the dynamic image of the structure in the centerright side and the six values of the strokes in the lower part.

If green, the led indicates that all strokes are in range; if red, it means at least one stroke exceeds the limits.

Then, the inverse kinematics subVI is integrated in the PTP control application, including the PI libraries (Fig. 11).

The HEXAGENT system can also be programmed to perform movements following a predefined trajectory using the parametric equations of that curve inside the workspace. Different programs were tested: linear motion, generation of a circular helix, generation of an elliptical helix, pivoting around a fixed point.

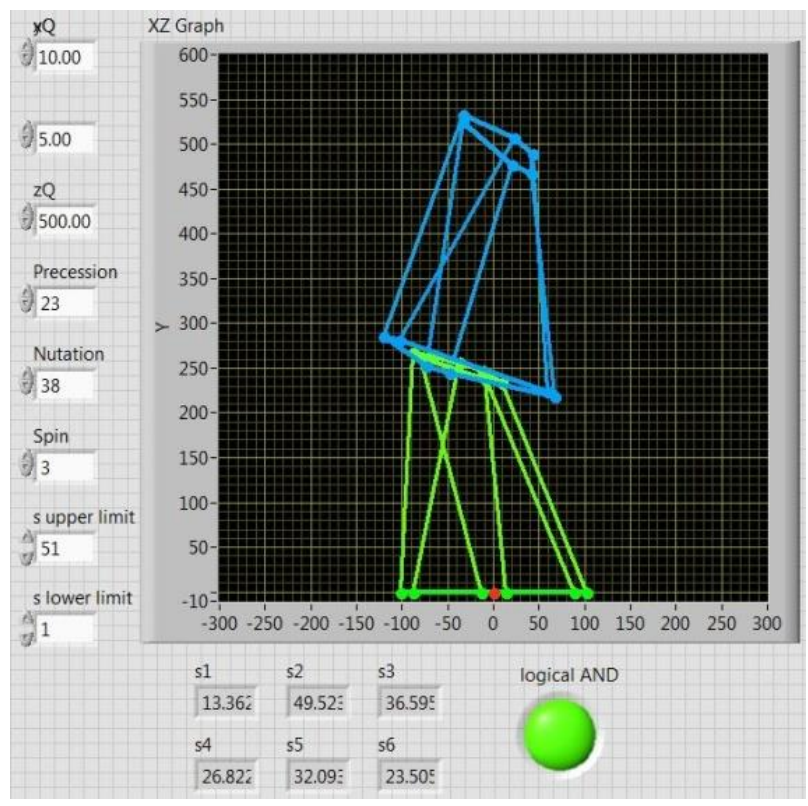

Fig. 10 HEXAGENT PTP Control - Control Panel

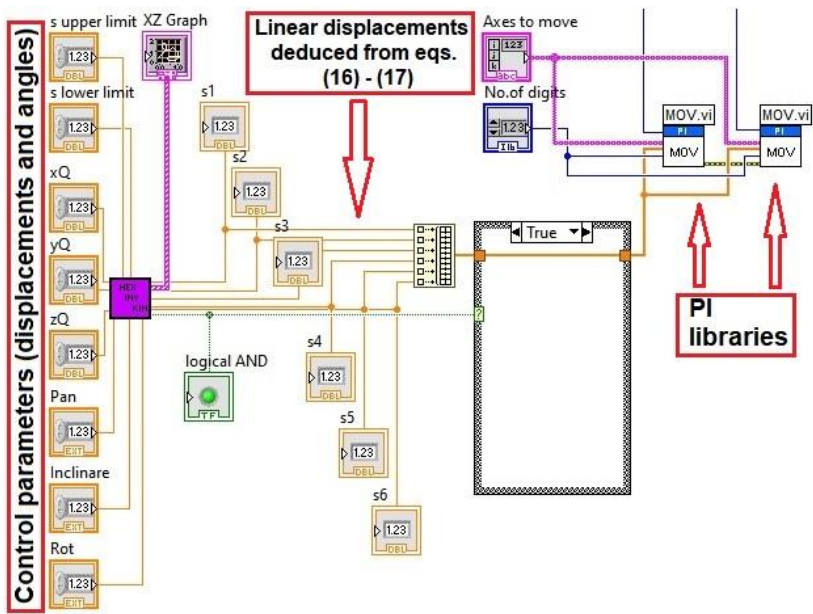

Fig. 11 PTP Control - Block Diagram - partial view

\section{Experimental details and results}

The aim of the demonstrator was to test its functionality and performances, especially in terms of accuracy and maneuverability. A special attention is paid to the double hexapod workspace. 


\subsection{Precision and repeatability}

As indicators of the overall accuracy criterion of the HEXAGENT system, precision and repeatability of the unidirectional positioning are used.

Measurements were performed with a Mahr digital comparator with resolution of $0.001 \mathrm{~mm}$ and stroke of 100 $\mathrm{mm}$, mounted on a Mitutoyo support (Fig. 12).

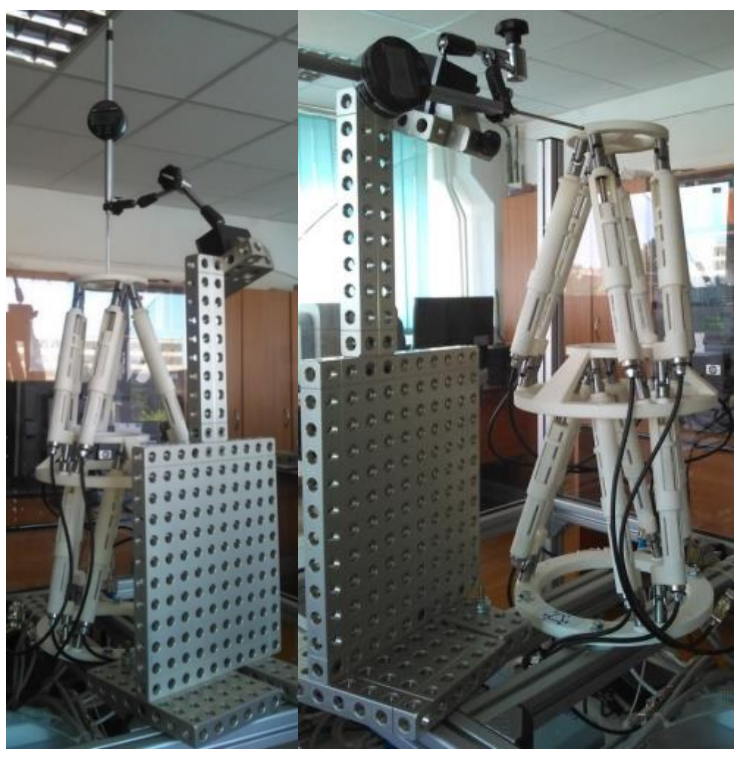

a

b

Fig. 12 Measuring precision and repeatability: $\mathrm{a}-\mathrm{z}$ direction; $\mathrm{b}-\mathrm{y}$ direction

For the $\mathrm{z}$ direction, the following values are obtained for the two parameters:

- positioning precision - $0.103 \mathrm{~mm}$;

- repeatability of unidirectional positioning $-0.021 \mathrm{~mm}$.

For the y direction, the following values are obtained for the same parameters:

- positioning precision $0.296 \mathrm{~mm}$;

- repeatability of unidirectional positioning $0.016 \mathrm{~mm}$.

As in the previous case, the precision performances are considered to be very good; the precision of approx. 0.3 $\mathrm{mm}$ indicates a systematic error that most likely comes from mounting and can be corrected by a calibration operation, and the repeatability of less than two hundredths of $\mathrm{mm}$ is even better than when moving in the $\mathrm{z}$ direction, although the stroke was higher.

For this demonstrator, the precision performances are considered to be very good, as expected. In an industrial construction, with the parts of the linear module executed by steel and tighter tolerances, it is expected to obtain a more accurate positioning.

\subsection{Double hexapod workspace}

In order to evaluate the workspace, two simulation programs of the workspaces by clouds of points were designed, - for one hexapod, respectively for a double hexapod: 1) Single Hexapod Workspace and 2) Double Hexapod Workspace.

With these programs, the workspaces for the single (Fig. 13, a) and for the double hexapod (Fig. 13, b) can be visualized and compared, with the observation that the cloud of points is generated for a given set of input angles.

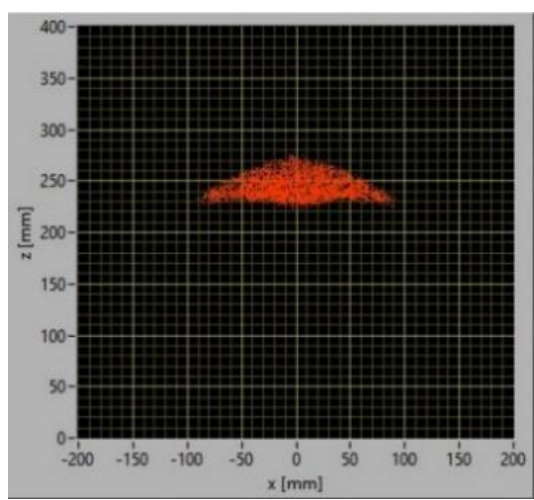

a

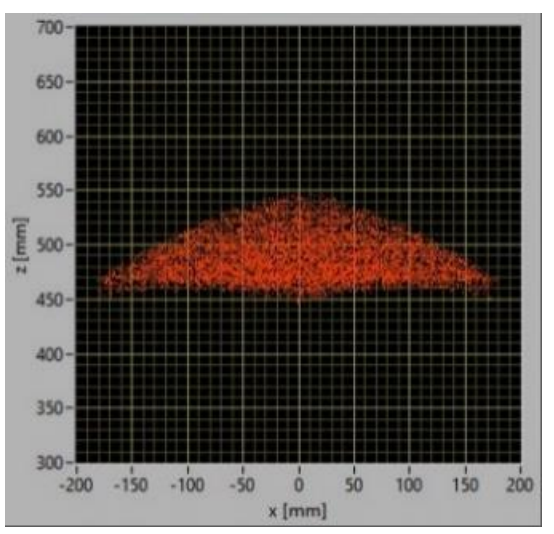

b

Fig. 13 The workspace for null rotations: a- for simple hexapod, b - for double hexapod

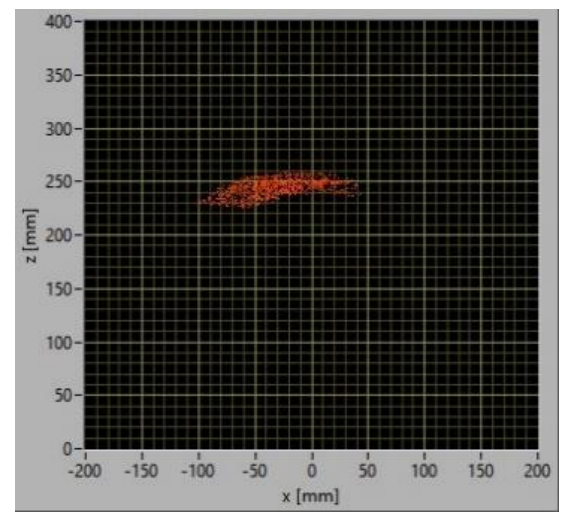

a

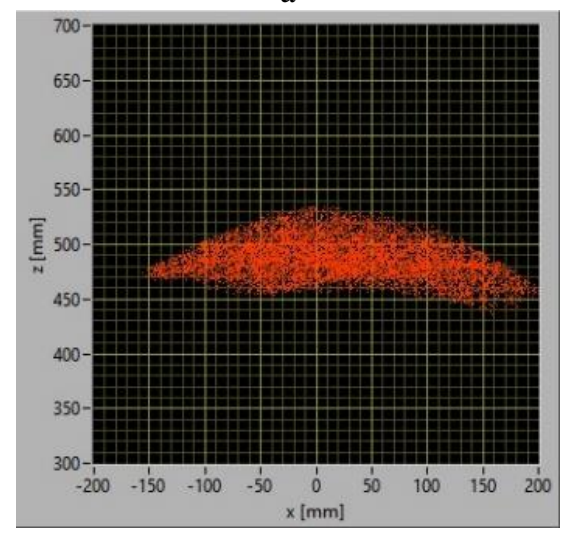

b

Fig. 14 The workspace for null precession and spin angles, but nutation angle equal to $20^{\circ}$ : a - for simple hexapod; $b$ - for double hexapod 
In the case presented above, with null rotations, the ratio of the two volumes is equal to $2^{3}=8$. A similar result is obtained for null precession and intrinsic rotation angles, but with the nutation angle equal to $20^{\circ}$ (Fig. 14).

The workspace of one hexapod depends on the ratio between the useful stroke and the length of the actuator. However, it must be noticed that possible collisions among the mechanical elements, difficult to be analytically expressed, may affect the theoretical workspace. On the other hand, the total workspace is obtained by reuniting the workspaces for various values of Euler's angles, which leads to a larger space than the one represented in the figures above.

\subsection{System maneuverability}

In order to test the system maneuverability, it was programmed to execute complex motions in extended workspaces compared with a single hexapod, such as multiple linear motions simulating a drilling operation, a circular helix and also an elliptical helix, by the aid of the HEXAGENT Elliptical Helix application software (Fig. 15).
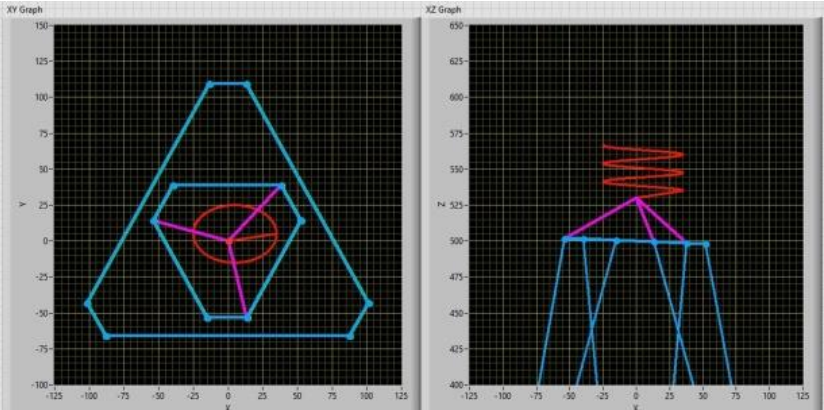

Fig. 15 Programming of movement on an elliptical helix path

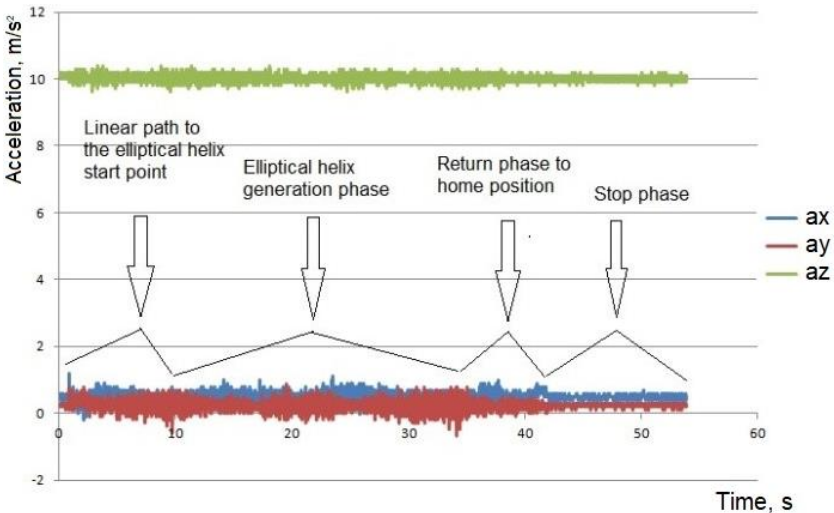

Fig. 16 Acceleration components indicating a smooth motion

The center of ellipse was moved in $x, y, z$ coordinates, also the parameters of the ellipse were modified. Furthermore, the tilt angle of the platform was changed in order to generate the same helix from a different angle. In all cases, the behavior of the demonstrator was considered adequate. The acceleration components obtained with a tri-axial accelerometer mounted on the upper platform indicate a smooth motion, with small peaks in the transition phases from a type of path to other (Fig. 16)

But the main advantage of this structure is it can perform these complex motions in an extended space compared with a single hexapod and in the same time it provides
$6 \mathrm{DOFs}$ at the final element being more compact and robust than a serial robot of comparable sizes (Fig. 17).

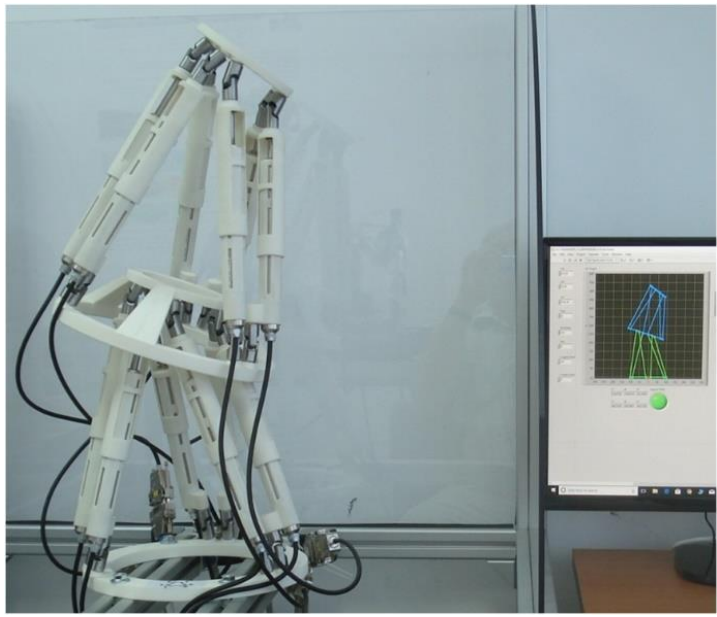

Fig. 17 HEXAGENT work position

\section{Discussion}

As it has been presented, the control of this double hexapod demonstrator works under the condition that the two hexapods have identical configurations at any moment. Removing this constraint, the system returns to the 12 DOFs which are physically possible, but it will be much more difficult to be controlled. Other constraints must be imposed in order to reduce its over redundancy.

Associated software applications were developed in order to evaluate the system workspace under different hypotheses. The limitations due to the maximum angle allowed by the universal joints are relatively easy to be implemented, but collision avoidance of the hexapod legs must be studied for every particular construction.

Using the PTP software, more precisely introducing the inverse kinematics algorithm in six For loops, corresponding to the six input values, the displacements $q_{i}$ of the actuators are computed. Selecting a vicinity around certain values of $q_{i}$ and saving both input and output data in an Excel file, were obtained maps for several zones of the 6dimensional space and thus a numerical solution of the direct kinematics, with a given accuracy. Considering vicinities converging to zero, more and more accurate $q_{i}$ values are obtained.

However, the biggest obstacle is the huge amount of data, which makes the process very time-consuming. With a standard PC or laptop, the number of iterations is equal to approx. $10^{6}$ per minute - not sufficient. For example, a billion of iterations are generated in 16.66 hours, and the resolution is still poor. A possibility is to suppress some DOFs and specially to restrain the space to zones of interest, which are mapped with increased resolution in an off-line process.

Self-learning algorithms could also be used to map the external coordinates to the internal coordinates by training and used further to control the robot.

On the other hand, the master-slave type control, performed by the aid of a $6 \mathrm{D}$ joystick is certainly very useful in many applications, possible in robotized surgery. To make the control more intuitive, the joystick could be also built as a hexapod, where actuators are replaced with displacement sensors. By construction, the device can assure 
the scaling of motion in order to improve the motion accuracy. In order to obtain the best results in terms of miniaturisation, the 6D control device could be based on flexible/ compliant joints, because they eliminate friction and backlash.

Moreover, two arms, each consisting of a double hexapod structure may be imagined, collaborating each other.

\section{Conclusions}

The HEXAGENT demonstrator contains several innovative solutions and proved an excellent functionality. The expected performances, as positioning accuracy and volume of the workspace, were also confirmed. The control software, based on the inverse kinematics of the double hexapod, is parameterized and can be easily adapted to any geometric characteristics - sizes and angles. The double hexapod structure may be used in different applications: special processing technologies or in complex, accurate and robust manipulations. Future researches regarding specific intelligent control algorithms could highlight new advantages and application possibilities.

\section{Acknowledgements}

This work, including the demonstrator, was supported by a grant of the Romanian National Authority for Scientific Research and Innovation, CNCS/ CCCDI UEFISCDI, project number PNIII-P2-2.1-PED-2016-0412, contract no. 50 PED /2017, within PNCDI III and also by a grant of the Romanian Ministry of Research and Innovation, CCCDI - UEFISCDI, project number PN-III- P1-1.2PCCDI-2017-0086 / contract no. 22 PCCDI /2018 within PNCDI III.

\section{References}

1. Raghavan, M. 1993. The Stewart platform of general geometry has 40 configurations, ASME J. Mechanical Design 115: 277-282.

2. Dietmaier, P. 1998. The Stewart-Gough platform of general geometry can have 40 real postures, Lenarcic, J., Husty, M.L. (Eds.), Advances in Robot Kinematics: Analysis and Control. Kluwer Academic Publishers, p. 7-16.

3. Merlet, J. P. 2006. Parallel Robots, 2nd ed., Springer, p. 63-64.

4. Lee, M. K.; Park, K. W. 1999. Kinematic and dynamic analysis of a double parallel manipulator for enlarging workspace and avoiding singularities, IEEE transactions on robotics and automation 15(6): 1024-1034. https://doi.org/10.1109/70.817667.

5. Lee, M. K.; Park, K. W. 2000. Workspace and singularity analysis of a double parallel manipulator, IEEE/ASME transactions on Mechatronics 5(4): 367375. https://doi.org/10.1109/3516.891048.
6. Mărgăritescu, M.; Moldovanu, A.; Roaţ, C.; Brişan, C. 2009. Aspects Concerning Virtual Models for a Double Hexapodal Platform, Annals of DAAAM for 2009 \& Proceedings of 20th DAAAM International Symposium, Vienna, November 25-28, 2009, ISSN 1726-9679, ISBN 978-3-901509-70-4, p. 423-424.

7. Rakhodaei, H.; Saadat, M. 2014. Inverse Dynamic and Control of a Hybrid Parallel Robot, Proceedings of the ASME 12th Biennial Conference on Engineering Systems design and Analysis ESDA2014, Copenhagen, Denmark.

8. Alimin, R.; Pasila, F. 2015. Design of Two-Serial Hexapod of Discrete Manipulator, Seventh International Conference on Computational Intelligence, Modelling and Simulation, Malaysia 36: 53-57. https://doi.org/10.1109/CIMSim.

9. Angeles, J. 1996. Fundamentals of Robotic Mechanical Systems: Theory, Methods and Algorithms, Second Edition, Springer.

10. Dudita, F.; Diaconescu, D.; Gogu, G. 1989. Articulated mechanisms. Inventics. Kinematics, Technical Publishing House, ISBN 973-31-0119-2, Bucharest (in Romanian).

11. Mărgăritescu, M.; Brişan, C.; Panaitopol, H. 2016. Multi Hexapod Robotic Positioning System, Romanian Patent OSIM no. 125589/ 2016 (in Romanian).

M. Mărgăritescu, D. Dumitriu, C. Brișan, A. M. E. Rolea, A. Constantin

\section{COMPLEX AND ROBUST MOTION PERFORMED IN EXTENDED WORKSPACE WITH A DOUBLE HEXAPOD ROBOTIC SYSTEM}

S u m m a r y

The aim of this study was to assess the functionality of a compact robotic system with hybrid kinematic structure, consisting in two hexapods serially connected and controlled in such a way that they have identical configurations any time, in terms of accuracy and maneuverability. By the aid of a demonstration model it was intended to prove that the system substantially benefits of the precision and robustness of the parallel structures and simultaneously is capable to generate complex motions in a significant extended workspace compared with a single hexapod. The double hexapod system answers the actual need of accurate, complex and robust positioning systems used in new technologies and possibly in robotic surgery.

Keywords: positioning system, Stewart platform, double hexapod, extended workspace.

Received July 18, 2018

Accepted December 01, 2020 